\title{
MicroRNA-155 modulates the proliferation of vascular smooth muscle cells by targeting endothelial nitric oxide synthase
}

\author{
JUN ZHANG, FEI ZHAO, XIAOLING YU, XIANG LU and GUOFENG ZHENG \\ Department of Respiratory Disease, The Fourth Affiliated Hospital of \\ China Medical University, Shenyang, Liaoning 110005, P.R. China
}

Received November 29, 2014; Accepted March 26, 2015

DOI: $10.3892 /$ ijmm.2015.2181

\begin{abstract}
A variety of microRNAs (miRNAs) have been reported to be significantly be involved in the regulation of vascular smooth muscle cell (VSMC) proliferation, which is an essential process for the formation of atherosclerotic plaque. The objective of the present study was to explore the role of microRNA-155 (miR-155) in the regulation of VSMC growth and migration. A total of 12 atherosclerotic plaque samples and 9 control samples were collected, and the expression levels of miR-155/endothelial nitric oxide synthase (eNOS) were determined in those samples by RT-qPCR and western blot analysis. The results revealed that the relative expression levels of miR-155 in the atherosclerotic plaque samples were significantly upregulated compared with those in the normal control samples. We further found eNOS to be an effective target of miR-155 in the VSMCs by luciferase assay, which was confirmed by the observation that VSMCs transfected with miR-155 mimics exhibited a significantly lower protein expression level of eNOS. We also demonstrated that the exogenous overexpression of miR-155 significantly enhanced cell proliferation by inhibiting apoptosis in human aortic SMCs (HASMCs), and it also promoted the migratory ability of the cells. In conclusion, our data demonstrate that miR-155 is significantly upregulated in atherosclerotic plaque, functioning to accelerate the proliferation and migration of VSMCs by targeting eNOS.
\end{abstract}

\section{Introduction}

Atherosclerosis is a common, complex, chronic disease with high morbidity and mortality, and it is characterized by the formation of atheromatous plaque in the intimal layer. A growing body of evidence indicates that the deregulation

Correspondence to: Professor Jun Zhang, Department of Respiratory Disease, The Fourth Affiliated Hospital of China Medical University, 102 Nanqi Road, Shenyang, Liaoning 110005, P.R. China E-mail: junzhang_resp@163.com

Key words: miR-155, vascular smooth muscle cell, endothelial nitric oxide synthase, atherosclerosis of cell behavior, such as proliferation and the migration of vascular smooth muscle cells (VSMCs) play essential roles in the pathogenesis of atherosclerosis (1). The formation of atheromatous plaque is believed to be initiated through the activation of endothelial cells by various metabolic risk factors, such as hyperlipidemia, hypertension or pro-inflammatory mediators. The aberrant activation of endothelial cells permits circulating monocytes to infiltrate the intima, leading to the formation of foam cells by the phagocytosis of low-density lipoprotein (LDL) cholesterol and oxidized phospholipids. Subsequently, atherosclerotic plaque is formed by proliferated VSMCs, physiologically located in the media of vessel walls, migrated to the intima (1-3). Migrated VSMCs in the intima can produce extracellular matrix (ECM), which is the major component of the fibrous cap of atherosclerotic plaque (4).

MicroRNAs (miRNAs or miRs) are a class of endogenous and small (approximately $22 \mathrm{nt}$ ) non-coding RNAs that suppress gene expression $\mathrm{BY}$ binding to the $3^{\prime}$ untranslated region (3'UTR) of mRNA transcripts to promote mRNA degradation or the translational inhibition of target mRNAs (5). To date, approximately 1,000 human miRNAs have been discovered and are believed to regulate up to $30 \%$ of gene expression, and are thereby involved in the regulation of a wide range of biological activities, including cell growth, apoptosis and differentiation. In recent years, accumulating evidence indicates that miRNAs play crucial roles in the control of VSMC function and the response to vascular injury by targeting transcriptional factors or key factors along certain signaling pathways in VSMC proliferation and migration (6,7). Indeed, a group of miRNAs, including miR-143/145, miR-221/222, miR-24, miR-26a, miR-1, miR-146a and miR-21, have been found to modulate VSMC differentiation, the phenotypic switch and neointimal formation under variable conditions (8-15).

Among these miRNAs, miR-155 is one of the most commonly studied miRNAs in the development of atherosclerosis $(16,17)$, and the downregulation of miR-155 has been reported to be involved in the susceptibility to atherosclerosis (18). Nevertheless, a previous study on miR-155 focused on its role in endothelial cells (19), and one of its target genes is endothelial nitric oxide synthase (eNOS); miR-155 downregulates eNOS expression by decreasing eNOS mRNA stability and by binding to its 3'UTR in human umbilical vein endothelial cells (HUVECs), and the knockdown of miR-155 prevents the cytokine-induced downregulation of eNOS expression, the 
reduction in nitric oxide (NO) production and the impairment of endothelium-dependent vascular relaxation (20). A previous study demonstrated that eNOS also plays a functional role in regulating the biological behaviors of VSMCs (21), which is consistent with other previous studies showing that the NO signaling pathway is involved in the regulation of the cellular activities of VSMCs $(22,23)$.

In the present study, we proved that miR-155 suppresses the expression level of eNOS by binding to the 3'UTR directly in VSMCs. Thus, it plays a significant role in the development of atherosclerosis.

\section{Materials and methods}

Sample collection. Samples were collected from atherosclerotic lesions from patients who received coronary artery bypass surgery or carotid endarterectomy $(n=12)$ and reference left internal mammary arteries were harvested during coronary artery bypass surgery $(n=9)$ at the Fourth Affiliated Hospital of China Medical University, Shenyang, China. Subjects with other cardiovascular diseases, such as aortic dissection and aneurysms were excluded from the study. The study protocol was approved by the Ethics Committee of China Medical University, and written informed consent was obtained from each participant prior to enrollment.

Cell culture. Human aortic SMCs (HASMCs) were cultured in SmGM-2 growth medium (both from Lonza, Walkersville, $\mathrm{MD}, \mathrm{USA}$ ) in $5 \%$ fetal bovine serum (FBS) following the manufacturer's instructions.

Isolation of RNA and reverse transcription-quantitative polymerase chain reaction $(R T-q P C R)$. Total RNA was isolated using the miRNeasy mini kit (Qiagen, Valencia, CA, USA) following the manufacturer's instructions. The quantity and quality of the RNA were then determined using the NanoDrop spectrophotometer (NanoDrop Technologies, Wilmington, DE, USA) and agarose electrophoresis. Subsequently, the cDNA of the target gene was synthesized using reverse transcriptase (Invitrogen, Carlsbad, CA, USA) and amplified using primer set, 5'-CTGTTAATGCTAATCGTGATAG-3' and 5'-GCAGGGTCCGAGGT-3' (miR-155); primer set, 5'-CTC GCTTCGGCAGCACA-3' and 5'-AACGCTTCACGAATTT GCGT-3' (U6); primer set, 5'-CCCTTCAGTGGCTGGT ACAT-3' and 5'-CACGATGGTGACTTTGGCTA-3' (eNOS). The SYBR-Green real-time detection system (from Bio-Rad Laboratories, Hercules, CA, USA) was used to quantify the signals. U6, an internal control, was used to normalize the miR-155 levels. The samples were all normalized to the endogenous control, U6. All fold changes were calculated using the $\Delta \Delta \mathrm{Ct}$ method.

Targeted miRNA overexpression. miR-115 mimics (5'-ttaaugct aatcgtgataggggt-3') and anti-eNOS siRNA (5'-tgtggaaagacaa ggcagca-3') were purchased from Ambion (Austin, TX, USA), and the VSMCs were transfected with high efficiency using the transfection Lipofectamine 2000 (Invitrogen). Successful transfection ( $>90 \%$ of all cells) was confirmed by visual fluorescent microscopic analysis. The in vitro experiments detailed below included a Cy3-labeled control scrambled oligo (nega- tive control) known to have no effect on any human miRNA to minimize the non-specific effect, and cohorts transfected with 30 or 100 pmol of miR-155 mimics, 100 pmol of anti-eNOS siRNAs or 40 ng pcDNA-eNOS.

Prediction of the target of $m i R-155$. We identified the potential target genes of miR-155 by searching the online microRNA database, http://targetscan.org/, and narrowed down the candidate genes by analyzing the pathological or physiological function of the candidate genes.

Boyden chamber chemotaxis assays. To evaluate the migratory capability of the VSMCs, a modified Boyden chamber assay was conducted, as previously described (24). Briefly, 6-well Transwell migration chambers with $8 \mu \mathrm{m}$ pores (Becton-Dickinson, Franklin Lakes, NJ, USA) were used, and 6x10 $10^{4}$ HASMCs transfected with miR-155 mimics, anti-eNOS siRNA, pcDNA-eNOS or the control were serum-starved for $24 \mathrm{~h}$ and then placed in the upper chamber in $1 \mathrm{ml}$ of medium. A total of $2 \mathrm{ml}$ of SmGM-2 medium were added to the lower chamber, and $24 \mathrm{~h}$ later, the migrated cells were fixed, stained with trypan blue and counted.

Proliferationand cell survivalassays. A modified 3-[4,5-dimethylthiazol-2-yl)-2,5-diphenyltetrazolium bromide (MTT) assay was performed to examine the HASMC viability, as previously described (25). Briefly, the HASMCs transfected with miR-155 mimics, anti-eNOS siRNAs or pcDNA-eNOS were cultured in 96-well plates for $24 \mathrm{~h}$ and then $10 \mu \mathrm{l}$ of MTT AB solution (Millipore, Billerica, MA, USA) were added, followed by incubation for $4 \mathrm{~h}$. The absorbance was determined at $570 \mathrm{~nm}$ (reference wavelength, $630 \mathrm{~nm}$ ).

Apoptosis assays. Programmed cell death rates were assessed using a commercially available apoptosis assay kit (Becton-Dickinson). The differentially transfected HASMCs were treated with $10 \% \mathrm{H}_{2} \mathrm{O}_{2}$ in serum-free medium for $6 \mathrm{~h}$. Subsequently, $1 \times 10^{5}$ cells were harvested and stained with $10 \mu \mathrm{l}$ FITC Annexin V and $10 \mu \mathrm{l}$ propidium iodide and FACS sorted within $1 \mathrm{~h}$ [BD FACSCalibur, $530 \mathrm{~nm}$ (FL1) and >575 nm (FL3); Becton-Dickinson].

Western blot analysis. The differentially treated HASMCs were harvested and lysed, and the cell lysates were subjected to $10 \%$ polyacrylamide gel electrophoresis (PAGE). The separated proteins were transferred onto a polyvinylidene difluoride (PVDF) membrane which was blocked with 5\% non-fat milk solution under room temperature for $1 \mathrm{~h}$. Subsequently, the PVDF membrane was incubated with primary antibodies directed against human eNOS (1:500; ab66127) and $\beta$-actin (1:1,000; ab6276) (Abcam, Cambridge, MA, USA), and an HRP-conjugated goat-anti-rabbit secondary antibody $(1: 2,000)$ (Cell Signaling Technology, Danvers, MA, USA). Exposed films were scanned and fluorescence signals were detected using the ECL kit (Applygen, Beijing, China) and integrated band densities were densitometrically analyzed with the background subtracted.

Luciferase assay. The full-length of human eNOS 3'UTR was PCR-amplified from the genomic DNA sample. The PCR product 

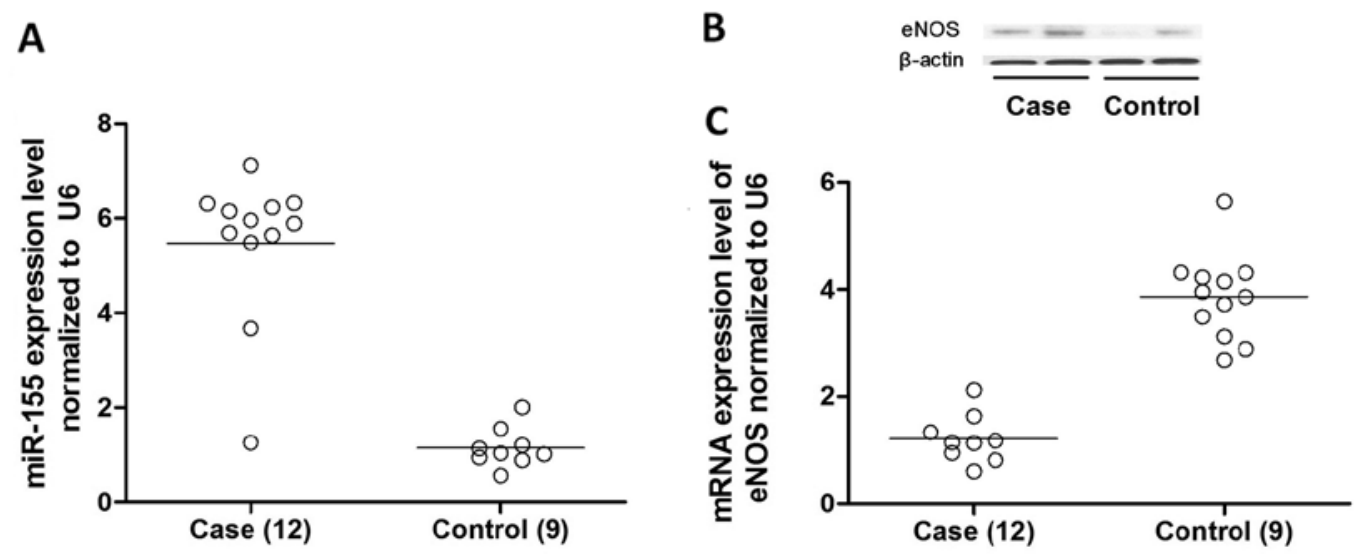

Figure 1. Expression levels of (A) miR-155, and (B) protein and (C) mRNA expression levels of endothelial nitric oxide synthase (eNOS) were determined in 12 sample tissues collected from atherosclerotic lesions from patients who received coronary artery bypass surgery or carotid endarterectomy and 9 control samples by RT-qPCR and western blot analysis.

Table I. Demographic parameters and clinical characteristics of the cases and controls enrolled in this study.

\begin{tabular}{lccc}
\hline Variables & $\begin{array}{c}\text { Controls } \\
(\mathrm{n}=9)\end{array}$ & $\begin{array}{c}\text { Cases } \\
(\mathrm{n}=12)\end{array}$ & P-value \\
\hline Age (years) & $61.4 \pm 6.3$ & $62.1 \pm 6.7$ & 0.811 \\
Gender (M/F) & $6 / 3$ & $9 / 3$ & 0.677 \\
Blood pressure (mmHg) & & & \\
$\quad$ Systolic BP & $142 \pm 11.6$ & $151 \pm 17.2$ & 0.192 \\
Diastolic BP & $92 \pm 10.2$ & $96 \pm 11.4$ & 0.416 \\
Glucose (mg/dl) & $138 \pm 14.8$ & $146 \pm 15.1$ & 0.241 \\
Body mass index $\left(\mathrm{kg} / \mathrm{m}^{2}\right)$ & $27.3 \pm 4.7$ & $28.5 \pm 4.8$ & 0.574 \\
\hline
\end{tabular}

was then cloned using the TA cloning kit (Invitrogen), and the accuracy of the insert was confirmed by Sanger sequencing. Subsequently, the QuickChange XL site-directed mutagenesis kit (Stratagene, La Jolla, CA, USA) was used to introduce the variant. Finally, the 3'UTR of Renilla luciferase in the vector pRL-SV40 (Promega, Madison, WI, USA) was substituted with the generated wild-type and mutant 3'UTR of eNOS. Luciferase assay was performed using the HASMCs, and the cells were seeded at $1 \times 10^{5}$ cells/well in 24-well plates. After $12 \mathrm{~h}$, the cells were transfected with miR-155 mimics together with pRL-SV40 containing the wild-type or mutant 3'UTR of eNOS using Lipofectamine 2000 (Invitrogen) according to manufacturer's instructions. Twenty-four hours after transfection, the cells were harvested by the addition of passive lysis buffer. Luciferase activities in the cell lysate were determined using the Dual-Luciferase assay system (Promega) on a TD-20/20 luminometer (Turner BioSystems, Sunnyvale, CA, USA).

Determination of the concentration of NO and cyclic guanosine monophosphate (cGMP) in HASMCs. The concentrations of NO and cGMP were determined in the HASMCs transfected with miR-155 mimics or anti-eNOS siRNA using the nitric oxide assay kit (Abcam) and the cGMP assay kit (Cell Signaling Technology).
Overexpression of eNOS. The coding sequence of eNOS was amplified using the following primers: 5'-ATAAGAATGCG GCCGCATGGGCAACTTGAAGAGCGTGGC-3' and 5'-GGT CTAGATCAGGGGCTGTTGGTGTCTGAGCC-3'. The PCR product was purified and inserted into the NotI and $\mathrm{XbaI}$ restrictive sites in pcDNA3.1. The accuracy of the insert was verified using direct Sanger sequencing. The plasmid containing eNOS was transfected into the HASMCs using Lipofectamine 2000.

Statistical analysis. Data are presented as the means \pm SD. All in vitro experiments included at least triple replicates per group. Data were subjected to the Kolmogorov-Smirnov test to determine distribution. Groups were compared using the two-tailed Student's t-test for parametric data (two groups comparison) or one-way ANOVA (multiple groups comparison). A value of $\mathrm{P}<0.05$ was considered to indicate a statistically significant difference. Data analysis was performed using SPSS 20.0 software (SPSS Inc., Chicago, IL, USA).

\section{Results}

Comparison of miR-155 between atherosclerotic lesions and normal control samples. Twelve samples from atherosclerotic lesions and 9 normal control samples were collected and the demographic parameters and clinical characteristics are presented in Table I. No difference was noted regarding the age, gender, body mass index (BMI), and blood pressure and blood glucose levels. The expression levels of miR-145, miR-221, miR-26a, miR-1, miR-146a, miR-21 and miR-155 were determined and compared between the 2 groups by RT-qPCR, and no difference was noted regarding the expression levels of these miRs (data not shown) except for miR-155. The relative expression of miR-155 in the atherosclerotic plaque samples was significantly upregulated to approximately $500 \%$ compared with normal controls (Fig. 1A).

eNOS is a direct target of miR-155 in VSMCs. Based on the computational analysis using an online target predicting tool (www.targetscan.org), we identified that miR-155 was able to bind to the 3'UTR of eNOS mRNA, indicating that this gene may be a potential molecular target of miR-155 (Fig. 2A). To 
A

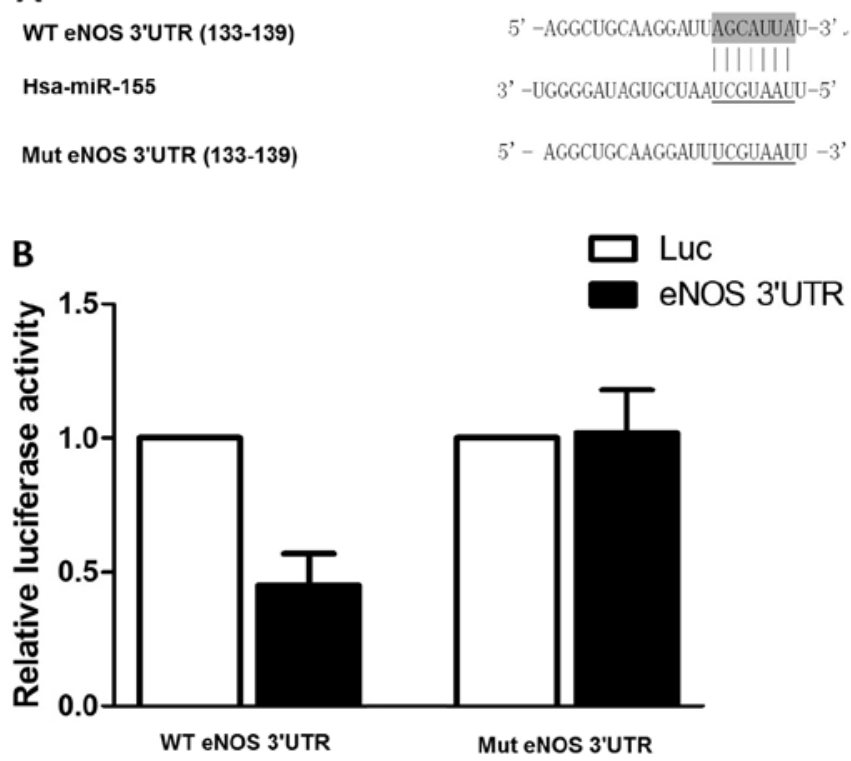

Figure 2. (A) Comparison between miR-155 and wild-type/mutant endothelial nitric oxide synthase (eNOS) 3' untranslated region (3'UTR). (B) The results of luciferase assay revealed that the luciferase activity in the cells transfected with miR-155 and wild-type eNOS 3'UTR was much lower than that of the cells transfected with miR-155 and mutant 3'UTR of eNOS.

determine whether miR-155 regulates the expression of eNOS by directly interacting with the $3^{\prime} \mathrm{UTR}$ of the gene in VSMCs, we subcloned the eNOS 3'UTR into a luciferase reporter plasmid and performed luciferase assay using the HASMCs. The results revealed that co-transfection of the miR-155 mimics with the eNOS 3'UTR reporter resulted in the inhibition of luciferase activity (Fig. 2B). However, miR-155 failed to suppress the activity of the mutant eNOS 3'UTR reporter (Fig. 2B). In agreement with the reporter assays, the overexpression of miR-155 following transfection with miR-155 mimics suppressed eNOS protein expression in a dose-dependent manner (Fig. 3A). These data indicate that eNOS is a direct target of miR-155.

miR-155 downregulates endogeneous eNOS expression in VSMCs by destabilizing eNOS mRNA. To further determine whether miR-155 suppresses eNOS expression in the VSMCs, we then examined the effects of miR-155 overexpression on the endogenous eNOS levels in the HASMCs transfected with miR-155 mimics. The overexpression of miR-155 for $48 \mathrm{~h}$ reduced the mRNA and protein expression levels of eNOS in the HASMCs in a dose dependent manner (Fig. 3). Importantly, the downregulation of eNOS expression correlated with the decrease in cGMP activity and NO production in the HASMCs (Fig. 4). The results revealed that the overexpression of miR-155 in the HASMCs significantly downregulated both the mRNA and protein expression levels of eNOS by promoting the degradation of eNOS mRNA.

Effects of miR-155 overexpression on VSMC proliferation. MTT assay was used to evaluate the proliferation of HASMCs, and HASMCs were transfected with either miR-155 mimics or the control prior to MTT assay. The results revealed that the exogenous expression of miR-155 significantly suppressed the

\section{A}

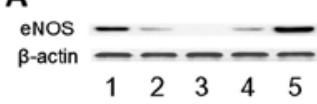

B

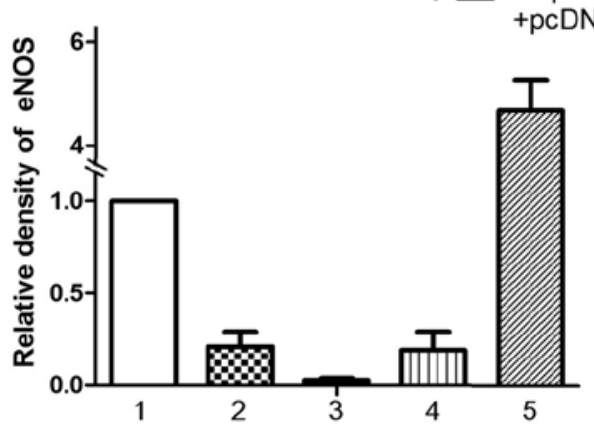

Figure 3. Protein expression level of endothelial nitric oxide synthase (eNOS) in the cells transfected with negative control (lane 1), 30 pmol miR-155 mimics (lane 2), 100 pmol miR-155 mimics (lane 3), 100 pmol anti-eNOS siRNA (lane 4) or 30 pmol miR-155 mimics + pcDNA-eNOS (lane 5) using (A) western blot analysis and corresponding densitometric analysis.

expression level of eNOS (Fig. 3A), and substantially promoted the proliferation of the HASMCs (Fig. 5A). Following incubation for $48 \mathrm{~h}$, a significantly enhanced effect on the proliferation of the transfected cells was observed compared with the controls, and the promoting rates were $250 \%(\mathrm{P}<0.01)$ for transfection with 100 pmol miR-155 mimics and $220 \%(\mathrm{P}<0.01)$ for transfection with 100 pmol anti-eNOS siRNAs, respectively, indicating that the overexpression of miR-155 promoted the proliferation of the HASMCs in vitro, and its effect was stronger than that of anti-eNOS siRNA. The ectopic overexpression of eNOS significantly reverse the increase in cell viability induced by transfection with miR-155 mimics and anti-eNOS siRNA (Fig. 5A).

Increased expression of miR-155 protects HASMCs against apoptosis. As eNOS functions as a pro-apoptotic protein and the exogenous expression of miR-155 causes a reduction in the enzyme, we then examined the effects of miR-155 on the apoptosis of HASMCs by flow cytometry. In the HASMCs, we found that transfection with miR-155 mimics and antieNOS siRNA similarly caused a reduction in the proportion of apoptotic and live cells (Fig. 5B-E). The ectopic overexpression of eNOS significantly reversed the decrease in apoptosis caused by transfection with miR-155 mimics and anti-eNOS siRNA (Fig. 5B-E).

Effects of miR-155 overexpression on VSMC proliferation and migration. To evaluate the role of miR-155 in the regulation of VSMC migration, we performed a Transwell migration assay. The results revealed that the exogenous expression of miR-155 significantly enhanced the migratory ability of the HCASMCs (Fig. 6A). Following incubation for $48 \mathrm{~h}$, a significantly enhanced effect on the migratory ability of the transfected cells was observed compared with the controls, and the promoting rates were $350 \%(\mathrm{P}<0.01)$ for transfection with 100 pmol miR-155 mimics and 210\% $(\mathrm{P}<0.01)$ for transfection 

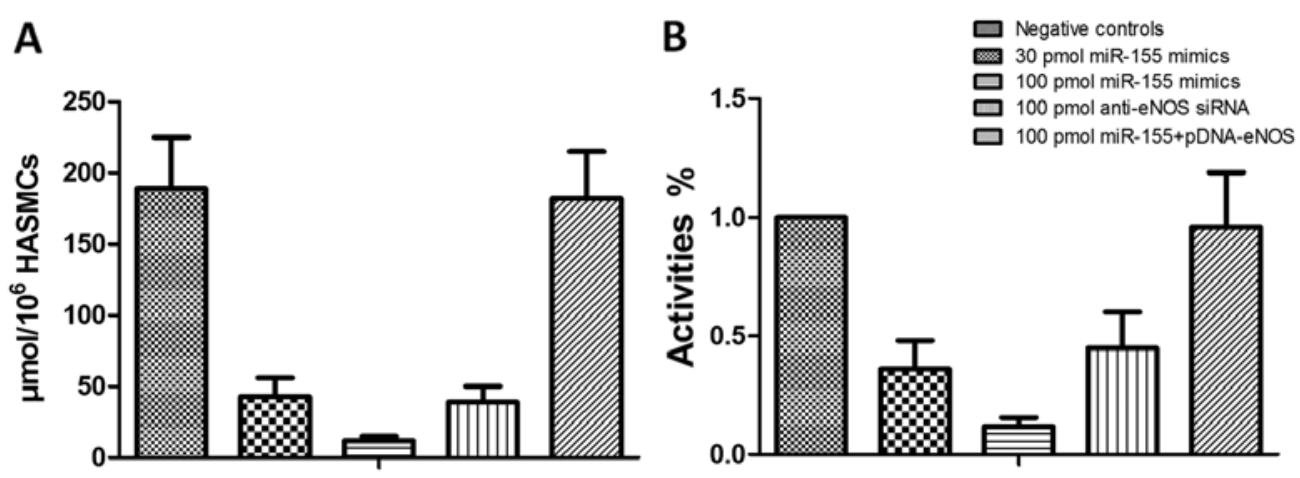

Figure 4. The production of (A) nitric oxide (NO) and (B) cyclic guanosine monophosphate (cGMP) in the human aortic SMCs (HASMCs) transfected with negative control and miR-155 mimics.

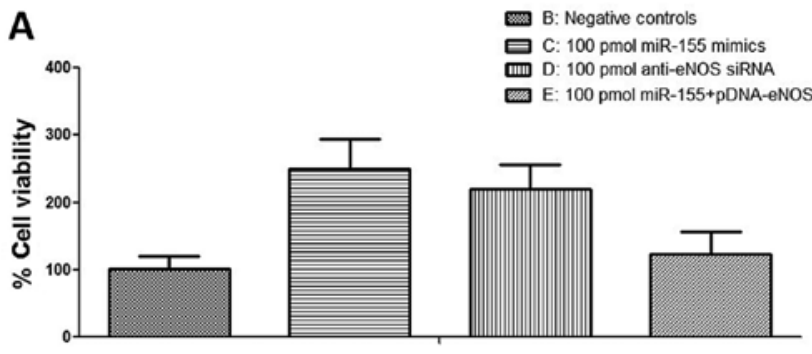

B

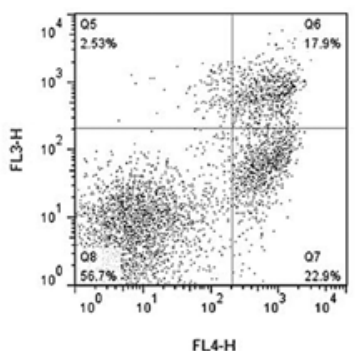

D

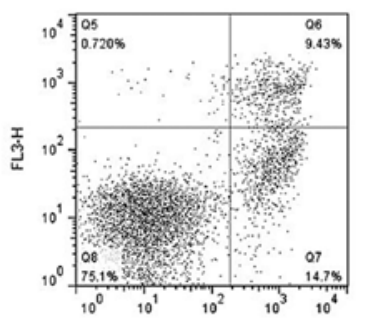

C

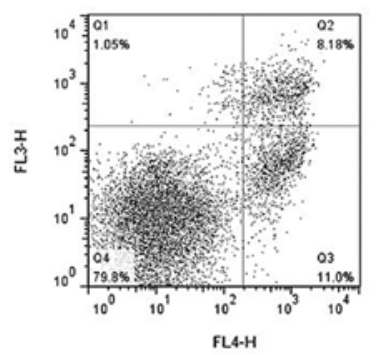

E

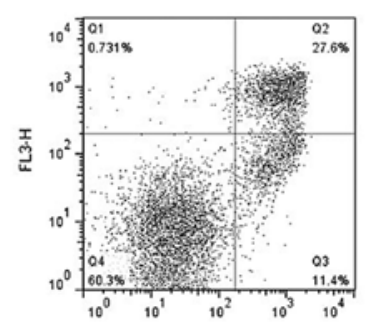

Figure 5. (A) Comparison of the proliferation of the human aortic SMCs (HASMCs) transfected with the negative control, 100 pmol miR-155 mimics, 100 pmol anti-endothelial nitric oxide synthase (eNOS) siRNA or 100 pmol miR-155 mimics + pcDNA-eNOS. Apoptosis was also evaluated in the HASMCs transfected with (B) negative control, (C) $100 \mathrm{pmol}$ miR-155 mimics, (D) 100 pmol anti-eNOS siRNA or (E) 100 pmol miR-155 mimics + pcDNA-eNOS.

with 100 pmol anti-eNOS siRNAs, respectively, indicating that the overexpression of miR-155 promotes the migratory ability of HASMCs in vitro, and that its effect was stronger than that of anti-eNOS siRNA. The ectopic overexpression of eNOS significantly reversed the increase in the migratory ability caused by transfection with miR-155 mimics and antieNOS siRNA (Fig. 6B-E).

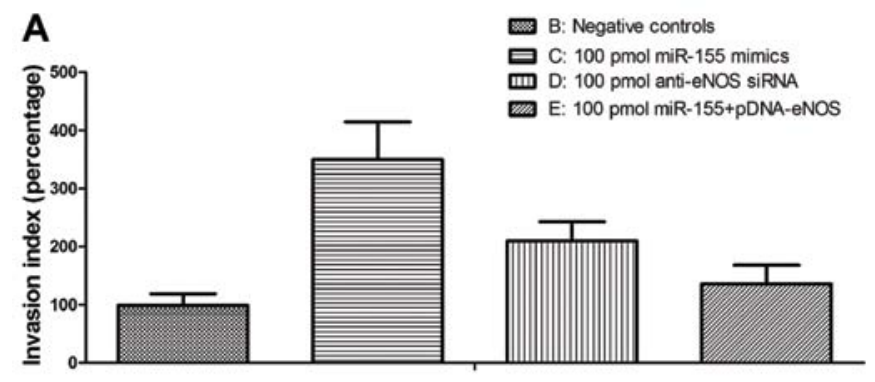

B

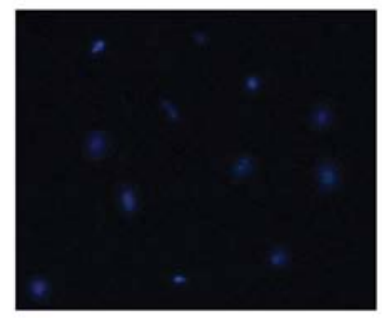

C

D

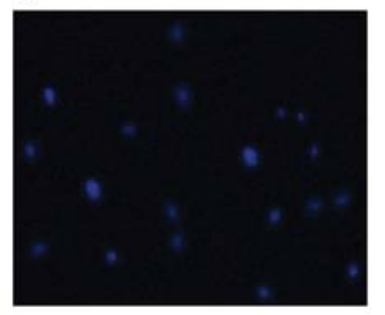

E

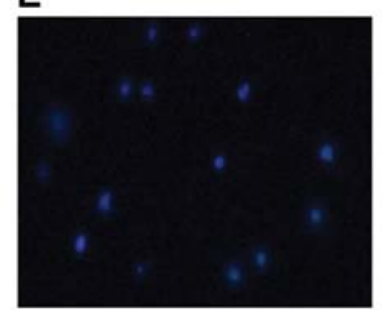

Figure 6. Comparison of the migratory abilities of (A) the human aortic SMCs (HASMCs) transfected with (B) negative control, (C) 100 pmol miR-155 mimics, (D) 100 pmol anti-endothelial nitric oxide synthase (eNOS) siRNA or (E) 100 pmol miR-155 mimics + pcDNA-eNOS.

\section{Discussion}

The proliferation and migration of VSMCs during the development of intimal hyperplasia are critical for the development of atherosclerotic lesions. In spite of rapid progress in our understanding of the role of miRNAs in VSMC biology (7), the molecular mechanism(s) underlying VSMC proliferation, migration, and proliferative vascular disease remain largely unknown. The present study identified miR-155 as a novel regulator of human VSMC proliferation and migration. We demonstrated that miR-155 is substantially upregulated in 
human atherosclerotic lesions and that the introduction of miR-155 significantly promoted the proliferation of VSMCs by inhibiting apoptosis. Additionally, the introduction of miR-155 promoted the migratory capability of the VSMCs. Furthermore, the ectopic overexpression of eNOS significantly reversed the increase in proliferation and in the migration of VSMCs caused by transfection with miR-155 mimics or anti-eNOS siRNAs. Thus, this study provides evidence implicating miR-155 as a novel key regulator in human VSMC biology.

The expression of miR-155 has been reported to be significantly upregulated in human and mouse atherosclerotic lesions, but circulating levels of miR-155 have been shown to be reduced in patients with coronary artery disease (17,26-28). The expression of miR-155 has been identified in VSMCs, endothelial cells and in activated macrophages, and thereby may affect most of the cell types involved in atherosclerosis (29-31). The majority of previous studies on miR-155 focused on its role in the activation of monocytes or macrophages during the development of atherosclerosis. It has been demonstrated that miR-155 is significantly upregulated in monocytes and dendritic cells in response to lipopolysaccharide (LPS) stimulation $(32,33)$ and during the pro-inflammatory activation of macrophages, a small group of miRNAs, including miR-155, is specifically upregulated $(29,34)$. In endothelial cells, miR-155 has been shown to be upregulated in response to a variety of stimuli, targeting the angiotensin II type 1 receptor and Ets-1, and reducing the pro-inflammatory activity of angiotensin II in endothelial cells (29). In VSMCs, the suppression of angiotensin II type 1 receptor by miR-155 may also attenuate the effects of angiotensin II on vascular remodeling (35). Data from animal experiments studying the effects of miR-155 on atherosclerosis are conflicting. Bone marrow cells are thought to be able to contribute to the formation of atherosclerotic plaque by relocating to the lesion and differentiating into VSMCs. It has been demonstrated that LDL-R $\mathrm{R}^{-/-}$mice carrying miR-155 $5^{-/}$bone marrow cells on a high-cholesterol diet developed slightly, but significantly more atherosclerosis. Whereas $\mathrm{ApoE}^{-/-}$mice harboring miR-155/- bone marrow cells had markedly less hypercholesterolemia-induced lesions compared with the controls $(28,36)$. Therefore, miR-155 may have different effects on lesion formation depending on the stage of atherosclerosis. In the present study, we confirmed the upregulation of miR-155 and the corresponding downregulation of its potential target, eNOS, in atherosclerotic lesions. Furthermore, eNOS was found to be a target gene of miR-155 in VSMCs by luciferase assay. The exogenous introduction of miR-155 substantially suppressed the expression of eNOS in the HASMCs, which could, at least partially, explain the proliferation- and migration-promoting effects of miR-155 in HASMCs.

NO produced by enzymatic reaction catalyzed by eNOS is an important homeostatic mechanism in the cardiovascular system. It has been reported that VSMC progenitor cells are recruited to injured arteries and significantly contribute to teh pathological neointimal VSMC accumulation in eNOS-deficient mice, and the same research group also demonstrated that eNOS deficiency increases the recruitment of mononuclear cells, including monocytes and lymphocytes into the arterial wall, and enhanced VSMC-rich neointimal lesion formation in a carotidartery ligation (CAL) model (21). These observations, together with those of previous studies showing that the NO signaling pathway is involved in the induction of the apoptosis of VSMCs $(22,23)$, led us to the hypothesis that the proliferation of VSMCs is accelerated by the deficiency of eNOS. To examine this hypothesis, the present study investigated the effects of eNOS deficiency, suppressed by the upregulation of miR-155, on the proliferation and migration of VSMCs, which are both critical for the formation of atherosclerotic plaque. Although the effects of eNOS/NO in cardiovascular systems have been investigated intensively, its role in the regulation of VSMCs in the cardiovascular system is only limitedly understood $(37,38)$. The formation of neointima seems to result from a combination of cellular infiltration and local proliferation, and the accumulation of VSMCs, migrated from the medial layer, at the site of atherosclerotic lesions may depend on the balance between apoptosis and proliferation. Our data demonstrated that eNOS deficiency, surppressed by miR-155, promoted cell proliferation by inhibiting the apoptosis of VSMCs. In addition to its ability to recruit circulating progenital cells into the lesion that then differentiate into smooth muscle cells, eNOS/NO deficiency also transports the VSMCs to the atheromatous lesion, and the lack of eNOS is also capable of inhibiting apoptosis and thereby accelerating the proliferation of VSMCs in situ. This result presented a new aspect of the vascular protective function of the eNOS/NO system and should be considered in interventions directed at VSMC accumulation in the neointimal lesion, which is a major component in the chain of events, causing the formation of atherosclerosis (39).

Taken together, the present study identified miR-155 as a novel regulator of human VSMCs by targeting, at least in part, the eNOS pathway. miR-155 expression was substantially upregulated in proliferative human VSMCs and we found that the restoration of eNOS expression markedly inhibited both VSMC proliferation and migration in response. This study provides significant novel insight into the molecular mechanisms associated with VSMC proliferation and migration, and suggests a potential therapeutic target for the prevention and treatment of human vascular diseases, such as atherosclerosis and restenosis.

\section{References}

1. Pidkovka NA, Cherepanova OA, Yoshida T, Alexander MR, Deaton RA, Thomas JA, Leitinger N and Owens GK: Oxidized phospholipids induce phenotypic switching of vascular smooth muscle cells in vivo and in vitro. Circ Res 101: 792-801, 2007.

2. Berliner JA and Watson AD: A role for oxidized phospholipids in atherosclerosis. N Engl J Med 353: 9-11, 2005.

3. Libby P, Ridker PM and Hansson GK: Progress and challenges in translating the biology of atherosclerosis. Nature 473: 317-325, 2011.

4. Lacolley P, Regnault V, Nicoletti A, Li Z and Michel JB: The vascular smooth muscle cell in arterial pathology: A cell that can take on multiple roles. Cardiovasc Res 95: 194-204, 2012.

5. Hata A: Functions of microRNAs in cardiovascular biology and disease. Annu Rev Physiol 75: 69-93, 2013.

6. Kang $\mathrm{H}$ and Hata A: MicroRNA regulation of smooth muscle gene expression and phenotype. Curr Opin Hematol 19: 224-231, 2012.

7. McDonald RA, Hata A, MacLean MR, Morrell NW and Baker AH: MicroRNA and vascular remodelling in acute vascular injury and pulmonary vascular remodelling. Cardiovasc Res 93: 594-604, 2012.

8. Cheng Y, Liu X, Yang J, Lin Y, Xu DZ, Lu Q, Deitch EA, Huo Y, Delphin ES and Zhang C: MicroRNA-145, a novel smooth muscle cell phenotypic marker and modulator, controls vascular neointimal lesion formation. Circ Res 105: 158-166, 2009. 
9. Cordes KR, Sheehy NT, White MP, Berry EC, Morton SU, Muth AN, Lee TH, Miano JM, Ivey KN and Srivastava D: miR-145 and miR-143 regulate smooth muscle cell fate and plasticity. Nature 460: 705-710, 2009.

10. Davis BN, Hilyard AC, Nguyen PH, Lagna G and Hata A: Induction of microRNA-221 by platelet-derived growth factor signaling is critical for modulation of vascular smooth muscle phenotype. J Biol Chem 284: 3728-3738, 2009.

11. Liu X, Cheng Y,Zhang S, Lin Y, Yang J and Zhang C: A necessary role of miR-221 and miR-222 in vascular smooth muscle cell proliferation and neointimal hyperplasia. Circ Res 104: 476-487, 2009.

12. Wang M, Li W, Chang GQ, Ye CS, Ou JS, Li XX, Liu Y, Cheang TY, Huang XL and Wang SM: MicroRNA-21 regulates vascular smooth muscle cell function via targeting tropomyosin 1 in arteriosclerosis obliterans of lower extremities. Arterioscler Thromb Vasc Biol 31: 2044-2053, 2011.

13. Chan MC, Hilyard AC, Wu C, Davis BN, Hill NS, Lal A, Lieberman J,Lagna $G$ and Hata A: Molecular basis for antagonism between PDGF and the TGFbeta family of signalling pathways by control of miR-24 expression. EMBO J 29: 559-573, 2010.

14. Ji R, Cheng Y, Yue J, Yang J, Liu X, Chen H, Dean DB and Zhang C: MicroRNA expression signature and antisense-mediated depletion reveal an essential role of microRNA in vascular neointimal lesion formation. Circ Res 100: 1579-1588, 2007.

15. Sun SG, Zheng B, Han M, Fang XM, Li HX, Miao SB, Su M, Han Y, Shi HJ and Wen JK: miR-146a and Krüppel-like factor 4 form a feedback loop to participate in vascular smooth muscle cell proliferation. EMBO Rep 12: 56-62, 2011.

16. Zhang E and Wu Y: Dual effects of miR-155 on macrophages at different stages of atherosclerosis: LDL is the key? Med Hypotheses 83: 74-78, 2014.

17. Tian FJ, An LN, Wang GK, Zhu JQ, Li Q, Zhang YY, Zeng A, Zou J, Zhu RF, Han XS, et al: Elevated microRNA-155 promotes foam cell formation by targeting HBP1 in atherogenesis. Cardiovasc Res 103: 100-110, 2014.

18. Hao L, Wang XG, Cheng JD, You SZ, Ma SH, Zhong X, Quan L and Luo B: The up-regulation of endothelin-1 and down-regulation of miRNA-125a-5p,-155, and -199a/b-3p in human atherosclerotic coronary artery. Cardiovasc Pathol 23: 217-223, 2014.

19. Weber M, Kim S, Patterson N, Rooney K and Searles CD MiRNA-155 targets myosin light chain kinase and modulates actin cytoskeleton organization in endothelial cells. Am J Physio Heart Circ Physiol 306: H1192-H1203, 2014.

20. Sun HX, Zeng DY, Li RT, Pang RP, Yang H, Hu YL, Zhang Q Jiang Y, Huang LY, Tang YB, et al: Essential role of microRNA-155 in regulating endothelium-dependent vasorelaxation by targeting endothelial nitric oxide synthase. Hypertension 60: 1407-1414, 2012.

21. Zhang LN, Wilson DW, da Cunha V, Sullivan ME, Vergona R, Rutledge JC and Wang YX: Endothelial NO synthase deficiency promotes smooth muscle progenitor cells in association with upregulation of stromal cell-derived factor-1alpha in a mouse model of carotid artery ligation. Arterioscler Thromb Vasc Biol 26: 765-772, 2006.

22. Boyle JJ, Weissberg PL and Bennett MR: Tumor necrosis factoralpha promotes macrophage-induced vascular smooth muscle cell apoptosis by direct and autocrine mechanisms. Arterioscler Thromb Vasc Biol 23: 1553-1558, 2003.

23. Gough PJ, Gomez IG, Wille PT and Raines EW: Macrophage expression of active MMP-9 induces acute plaque disruption in apoE-deficient mice. J Clin Invest 116: 59-69, 2006.
24. Ishida N, Hayashi K, Hattori A, Yogo K, Kimura T and Takeya T: CCR 1 acts downstream of NFAT2 in osteoclastogenesis and enhances cell migration. J Bone Miner Res 21: 48-57, 2006.

25. Erl W, Hristov M, Neureuter M, Yan ZQ, Hansson GK and Weber PC: HMG-CoA reductase inhibitors induce apoptosis in neointima-derived vascular smooth muscle cells. Atherosclerosis 169: 251-258, 2003.

26. Fichtlscherer S, De Rosa S, Fox H, Schwietz T, Fischer A, Liebetrau C, Weber M, Hamm CW, Röxe T, Müller-Ardogan M, et al: Circulating microRNAs in patients with coronary artery disease. Circ Res 107: 677-684, 2010.

27. Raitoharju E,Lyytikäinen LP,Levula M, Oksala N, Mennander A, Tarkka M, Klopp N, Illig T, Kähönen M, Karhunen PJ, et al: miR-21, miR-210, miR-34a, and $\mathrm{miR}-146 \mathrm{a} / \mathrm{b}$ are up-regulated in human atherosclerotic plaques in the Tampere Vascular Study. Atherosclerosis 219: 211-217, 2011.

28. Nazari-Jahantigh M, Wei Y, Noels H, Akhtar S, Zhou Z, Koenen RR, Heyll K, Gremse F, Kiessling F, Grommes J, et al: MicroRNA-155 promotes atherosclerosis by repressing Bcl6 in macrophages. J Clin Invest 122: 4190-4202, 2012.

29. Martin MM, Buckenberger JA, Jiang J, Malana GE, Nuovo GJ, Chotani M, Feldman DS, Schmittgen TD and Elton TS: The human angiotensin II type 1 receptor $+1166 \mathrm{~A} / \mathrm{C}$ polymorphism attenuates microRNA-155 binding. J Biol Chem 282 24262-24269, 2007.

30. O'Connell RM, Taganov KD, Boldin MP, Cheng G and Baltimore D: MicroRNA-155 is induced during the macrophage inflammatory response. Proc Natl Acad Sci USA 104: 1604-1609, 2007.

31. Zhu N, Zhang D, Chen S, Liu X, Lin L, Huang X, Guo Z, Liu J, Wang Y, Yuan W, et al: Endothelial enriched microRNAs regulate angiotensin II-induced endothelial inflammation and migration. Atherosclerosis 215: 286-293, 2011.

32. Ceppi M, Pereira PM, Dunand-Sauthier I, Barras E, Reith W, Santos MA and Pierre P: MicroRNA-155 modulates the interleukin-1 signaling pathway in activated human monocyte-derived dendritic cells. Proc Natl Acad Sci USA 106: 2735-2740, 2009.

33. Taganov KD, Boldin MP, Chang KJ and Baltimore D: NF-kappaB-dependent induction of microRNA miR-146, an inhibitor targeted to signaling proteins of innate immune responses. Proc Natl Acad Sci USA 103: 12481-12486, 2006.

34. Graff JW, Dickson AM, Clay G, McCaffrey AP and Wilson ME: Identifying functional microRNAs in macrophages with polarized phenotypes. J Biol Chem 287: 21816-21825, 2012.

35. Heeneman S, Sluimer JC and Daemen MJ: Angiotensinconverting enzyme and vascular remodeling. Circ Res 101: 441-454, 2007.

36. Donners MM, Wolfs IM, Stöger LJ, van der Vorst EP, Pöttgens CC, Heymans S, Schroen B, Gijbels MJ and de Winther MP: Hematopoietic miR155 deficiency enhances atherosclerosis and decreases plaque stability in hyperlipidemic mice. PLoS One 7: e35877, 2012

37. Naseem KM: The role of nitric oxide in cardiovascular diseases. Mol Aspects Med 26: 33-65, 2005.

38. Albrecht EW, Stegeman CA, Heeringa P, Henning RH and van Goor $\mathrm{H}$ : Protective role of endothelial nitric oxide synthase. J Pathol 199: 8-17, 2003.

39. Casscells W: Migration of smooth muscle and endothelial cells. Critical events in restenosis. Circulation 86: 723-729, 1992. 